\title{
Orthodontic treatment has little to do with temporomandibular disorders
}

\author{
Does traditional orthodontic treatment change the prevalence of \\ temporomandibular disorder?
}

\section{Kim MR, Graber TM, Viana MA. Orthodontics and temporomandibular disorder: a meta-analysis. Am J Orthod Dentofac Orthop 2002;121:438-446}

Data sources Studies were sourced using Medline, reference lists of identified articles and a list of published and unpublished articles compiled by RG Behrents (Professor and head of orthodontics at St. Louis University).

Study selection For inclusion in the review, an article had to satisfy the following criteria: orthodontic treatment was completed in each patient; clinical temporomandibular disorder (TMD) evaluation was performed in each patient (including at least one clinical evaluation after treatment); it was written in English; and the study type was not a case report or an 'opinion' paper.

Data extraction and synthesis Data were divided and extracted using a standardised form, according to study designs, symptoms, signs or indices. A statistical test for the hypothesis of parametric homogeneity was carried out, along with sensitivity analyses.

Results The search identified 960 articles, of which 31 met the inclusion criteria (18 cross-sectional studies or surveys and 13 longitudinal studies). Because of substantial heterogeneity, the results were summarised without further statistical analysis. No study indicated that traditional orthodontic treatment or the use of a specific appliance increased the prevalence of TMD, except for mild or transient signs, and only one article showed that extraction during orthodontic treatment changed the prevalence of TMD.

Conclusions Because the cause of TMD is not known, methodological shortcomings and lack of a widely accepted classification scheme, definitive conclusions cannot be drawn. The data, however, do not indicate that traditional orthodontic treatment increases the prevalence of TMD. It is clear that a reliable and valid diagnostic classification system for TMD is needed for future research.
Address for correspondence: Thomas M Graber, Department of Orthodontics (MC842) College of Dentistry, University of Illinois at Chicago, 801 South Paulina Street, Chicago IL 60612-7011, USA. E-mail: tgraber@uic.edu

\section{Commentary}

The current evidence in the literature suggests that there is no direct link between orthodontic treatment and TMD. Orthodontists are nevertheless occasionally blamed for TMD after treatment. In the late 1980s, a high-profile lawsuit in the US startled the orthodontic community and, as a result, many good studies were conducted and published to better understand the relationship between treatment and impact on the temporomandibular joint. Many reviews have been published for the busy practitioner and although some are well designed, they have often been biased because of the lack of a formal methodology.

This study is a meta-analysis based on the current clinical literature relating to orthodontic treatment and TMD. The authors must be congratulated for reviewing the available literature in a systematic manner, and for providing answers to relevant clinical questions. These can be summarized: does orthodontic treatment, the use of a specific appliance or extraction during orthodontic treatment, change the prevalence of TMD?

This extensive literature review identified 960 articles of which only 31 met the study's inclusion criteria for data extraction. The information was further divided according to study design (18 cross-sectional and 13 longitudinal) and clinical presentation (signs, symptoms or indexes). None of the studies reviewed indicated that traditional orthodontics (fixed, removable or functional appliances) increase the prevalence of TMD (except for mild signs). In addition, there was no correlation between orthodontic appliances and an increase in these disorders.

Importantly, the paper revealed that the outcome measures used to evaluate TMD were unreliable and there was a lack of consensus in reporting. As a result of the diversity in outcome measures, a true meta-analysis was not carried out: perhaps the greatest weakness of the study. This, however, highlights the need to standardise the reporting of clinical information with regards to orthodontic treatment and TMD in future studies.

Therefore, the question, "is there a relationship between orthodontic treatment and TMD?" has a cautious "No" as the answer, based on current evidence, The evidence on which we base our foundation needs to be improved to better understand the aetiology of TMD and to ensure that any risks involved, no matter how small, are reduced when patients are treated.

\section{Practice point}

- Current evidence shows no definite link between orthodontics and TMD but more research is required.

\footnotetext{
Chung Kau How

Evidence-Based Dentistry (2004) 5, 75.

doi:10.1038/sj.ebd.6400269
}

Dental Health and Biological Sciences, University of Wales College of Medicine Dental School, Cardiff, Wales, UK 